\title{
Ice Recrystallization Inhibition Is Insufficient to Explain Cryopreservation Abilities of Antifreeze Proteins
}

Yuling Sun, Daria Maltseva, Jie Liu, Theordore Hooker, II, Volker Mailänder, Hans Ramløv, Arthur L. DeVries, Mischa Bonn, and Konrad Meister*

Cite This: https://doi.org/10.1021/acs.biomac.1c01477

Read Online

\section{ACCESS |}

山lll Metrics \& More | 回 Article Recommendations

Supporting Information

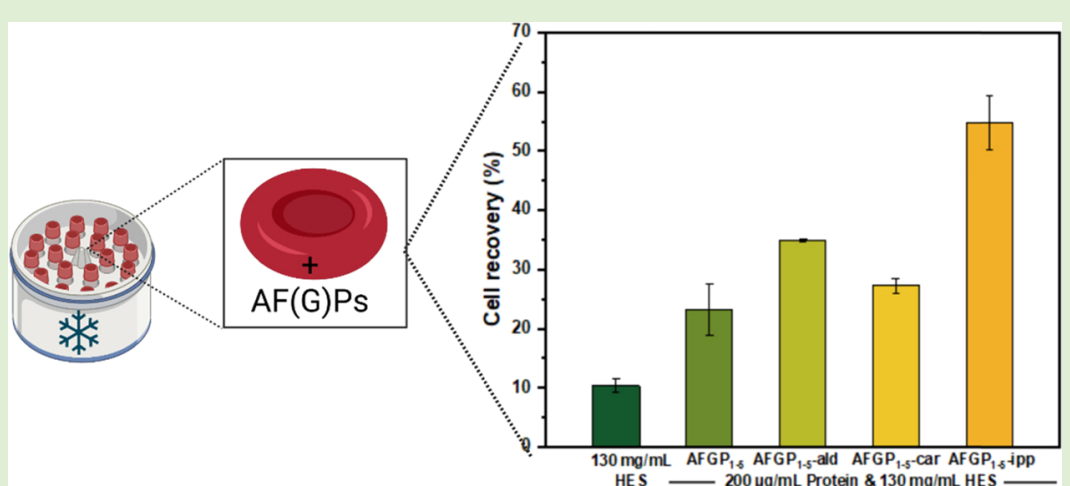

ABSTRACT: Antifreeze proteins (AFPs) and glycoproteins (AFGPs) are exemplary at modifying ice crystal growth and at inhibiting ice recrystallization (IRI) in frozen solutions. These properties make them highly attractive for cold storage and cryopreservation applications of biological tissue, food, and other water-based materials. The specific requirements for optimal cryostorage remain unknown, but high IRI activity has been proposed to be crucial. Here, we show that high IRI activity alone is insufficient to explain the beneficial effects of $\mathrm{AF}(G)$ Ps on human red blood cell (hRBC) survival. We show that $\mathrm{AF}(G) \mathrm{Ps}$ with different IRI activities cause similar cell recoveries of hRBCs and that a modified AFGP variant with decreased IRI activity shows increased cell recovery. The AFGP variant was found to have enhanced interactions with a hRBC model membrane, indicating that the capability to stabilize cell membranes is another important factor for increasing the survival of cells after cryostorage. This information should be considered when designing novel synthetic cryoprotectants.

\section{INTRODUCTION}

The transfusion of human red blood cells (hRBCs) is oftentimes the only option for patients suffering from leukemia, anemias, or traumas with severe blood loss. ${ }^{1,2}$ Long-term storage of RBCs is further crucial for the storage of rare blood cells, military transfusions, or special autotransfusion programs. ${ }^{3}$ Cryopreservation still remains the only method that allows long-term storage of hRBCs and access to quantities of $\mathrm{hRBC}$ units that are necessary when large numbers of hRBC transfusions are required. ${ }^{4}$ Unwanted ice formation and recrystallization during thawing present major challenges for cryopreserved biological samples and cause cellular damage of tissues and hRBC apoptosis. ${ }^{5,6}$ The clinical strategies for cryopreservation require the addition of high concentrations of cell-permeating cryoprotectants such as water-miscible organic solvents (e.g., glycerol and dimethyl sulfoxide). ${ }^{7-9}$ However, the high concentration of cryoprotectants and the toxicity of solvents require time-consuming removal of traces of toxic solvents, conflicting with rapid transplant or transfusions. Furthermore, the current cryopre- servation protocols do not effectively control extracellular ice growth, leaving tissues vulnerable to cellular damage.,10,11 Organisms inhabiting freezing environments and prone to ice recrystallization (IRI) injuries have evolved biomolecular solutions to enable life to flourish under icy conditions. ${ }^{12-14}$ They produce antifreeze proteins (AFPs) and glycoproteins (AFGPs) that have the ability to inhibit IRI, ${ }^{15}$ the ability to shape ice crystals into unusual morphologies, ${ }^{16}$ and the ability to depress the freezing point in a noncolligative manner (thermal hysteresis, TH). ${ }^{17}$ The relative magnitude of each effect varies between individual $\mathrm{AF}(G)$ Ps, with the AFGPs being the by far most potent IRI inhibitors. ${ }^{16,18}$ High IRI activity has been suggested to be key for optimal

Received: November 11, 2021

Revised: January 11, 2022 

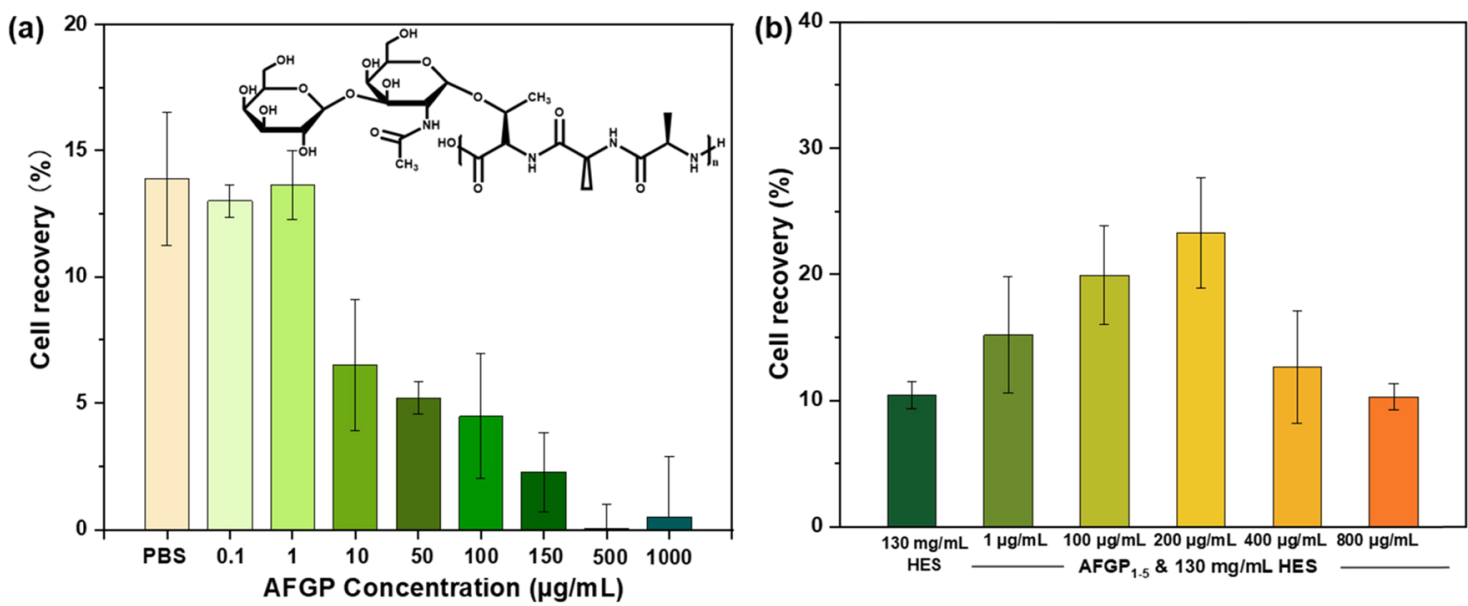

Figure 1. Cryopreservation of $\mathrm{hRBC}$ in the presence of $\mathrm{AFGP}_{1-5}$. (a) Recovery of hRBC cryopreserved in PBS solutions with different concentrations of $\mathrm{AFGP}_{1-5}$ thawed at $45{ }^{\circ} \mathrm{C}$. (b) Recovery of hRBC cryopreserved in HES solutions $(130 \mathrm{mg} / \mathrm{mL})$ with different AFGP $1-5$ concentrations thawed at $23{ }^{\circ} \mathrm{C}$. Experiments were performed three times, and the error bars represent the standard deviation between the individual measurements.

cryopreservation effects, and substantial efforts have been made to develop synthetic compounds that mimic $\operatorname{AF}(G)$ Ps IRI properties to increase cellular survival. ${ }^{5,10,19,20}$ However, up to now, the most active synthetic IRI compounds are substantially less active than their natural counterparts, and antifreeze mimics are rarely used in real-life applications. Recently, numerous studies have reported hydroxyethyl starch (HES) as a promising nonvitrifying cryoprotectant of RBCs, ${ }^{21,22}$ peripheral blood stem cells, ${ }^{23,24}$ keratinocytes, ${ }^{25-27}$ fibroblast, ${ }^{26}$ and other cell types. ${ }^{28,29}$ When used at appropriate concentrations, HES appears free of side effects and is less toxic than the commonly used cryoprotectants. ${ }^{30-32}$ However, transfusions containing a high concentration of HES can also have unwanted side effects like the inhibition of hemostasis in vulnerable patients. ${ }^{33,34}$

The usage of $\operatorname{AF}(G)$ Ps to protect RBCs has been shown to reduce the destruction of RBCs, hemolysis, after cryopreservation. ${ }^{35}$ Carpenter et al. showed that upon the addition of AFP I to RBCs cryopreserved in HES, their recovery due to the inhibition of IRI was enhanced. ${ }^{11}$ Interestingly, it was further reported that AFPs type I, II, and III reduced hemolysis at micromolar concentrations while enhancing hemolysis at AFP millimolar concentrations. ${ }^{36}$ The origin of this discrepancy, the specific requirements for optimized cryopreservation of $\mathrm{AF}(G) P s$ and mimics, and the role of IRI activity in cryopreservation remain debated. Here, we investigate the effects of different $\operatorname{AF}(G)$ Ps and variants on the survival of hRBC after cryostorage. Our results reveal that IRI activity alone is insufficient to explain the cell recovery after cryopreservation and that favorable protein-membrane interactions seem important.

\section{MATERIALS AND METHODS}

Human RBCs Preparation. Human blood was collected from healthy donors and centrifuged $\left(1940 \mathrm{~g}, 5 \mathrm{~min}, 25^{\circ} \mathrm{C}\right)$. The top layer (buffy coat and plasma) was removed and replaced with an equal volume of PBS buffer (Dulbecco's phosphate-buffered saline, $1 \times$, without calcium and magnesium). The RBCs were then washed three times with PBS buffer using identical conditions. The final PBS solution of RBCs had a packed cell volume of $\sim 40 \%$. Human blood was obtained from the Department of Transfusion Medicine Mainz from 10 healthy donors after physical examination and after obtaining their informed consent in accordance with the Declaration of
Helsinki. The use of human blood was approved by the local ethics committee "Landesärztekammer Rheinland-Pfalz" (837.439.12 (8540F)).

AFGP $_{1-5}$ was purified from the Antarctic toothfish Dissostichus mawsoni, as described previously. ${ }^{28}$ The $\mathrm{AFGP}_{1-5}$ variants were made by established protocols, as described recently. ${ }^{37}$ AFP I was purified from winter flounder, Pseudopleuronectes americanus. RmAFP was obtained by recombinant protein expression, as described elsewhere. Quaternary aminoethyl (QAE) (AFP-III) was purified from the Antarctic eelpout (Lycodichthys dearborni) or obtained by recombinant protein expression, including the mutant $\mathrm{T} 18 \mathrm{~N}$. In this mutant, the threonine residue at position 18 is replaced by asparagine, which causes the complete loss of antifreeze activity.

Cryopreservation of RBCs. Freshly prepared human RBCs (50 $\mu \mathrm{L}$ ) (packed cell volume $\sim 40 \%$ ) were added to $50 \mu \mathrm{L}$ of cryoprotectant $\left(\mathrm{AFGP}_{1-5}, \mathrm{AF}(G) \mathrm{Ps} / \mathrm{HES}\right.$, or $\mathrm{AF}(G)$ Ps variants/ HES) in cryovials and was gently mixed using a vortex. Triplicate independent samples were characterized for each group. All samples were rapidly frozen in liquid nitrogen and stored in liquid nitrogen for $20 \mathrm{~min}$. Samples were thawed for $10 \mathrm{~min}$ in a $45^{\circ} \mathrm{C}$ water bath or for $20 \mathrm{~min}$ at room temperature $\left(22 \pm 1{ }^{\circ} \mathrm{C}\right)$.

Control samples ( $0 \%$ hemolysis) were prepared by adding $50 \mu \mathrm{L}$ of freshly prepared hRBC to PBS buffer and storing them for $1 \mathrm{~h}$ at room temperature. Control samples ( $100 \%$ hemolysis) were prepared by adding $50 \mu \mathrm{L}$ of freshly prepared hRBC to $50 \mu \mathrm{L}$ of milli-Q water, and samples were vortexed. The $100 \%$ hemolysis samples were rapidly frozen in liquid nitrogen and stored in liquid nitrogen for $20 \mathrm{~min}$. Samples were thawed for $10 \mathrm{~min}$ in a $45^{\circ} \mathrm{C}$ water bath or for $20 \mathrm{~min}$ at room temperature $\left(23 \pm 1{ }^{\circ} \mathrm{C}\right)$.

Measurement of RBC Hemolysis and Cell Recovery. Sixty microliters of $\mathrm{RBC} /$ cryoprotectant suspension were added into 540 $\mu \mathrm{L}$ of PBS solution and centrifuged $\left(500 \mathrm{~g}, 5 \mathrm{~min}, 4^{\circ} \mathrm{C}\right)$. Thereafter, $200 \mu \mathrm{L}$ of the supernatant was removed and added into $3.8 \mathrm{~mL}$ of PBS solution. Absorbance was measured with an ultraviolet/visible (UV/vis) spectrometer (Lambda 900, Perkin Elmer) at $414 \mathrm{~nm}(1 \mathrm{~cm}$ light path). Hemolysis (\%) and cell recovery (\%) were calculated according to eqs 1 and 2, respectively. Triplicate samples were characterized for each group.

$$
\begin{aligned}
\operatorname{Hemolysis}(\%)= & \frac{\text { Abs }- \text { Abs (0\%hemolysis })}{\text { Abs (100\%hemolysis })- \text { Abs (0\%hemolysis) }} \\
& * 100
\end{aligned}
$$

Cell recovery $(\%)=100(\%)-$ hemolysis $(\%)$ 

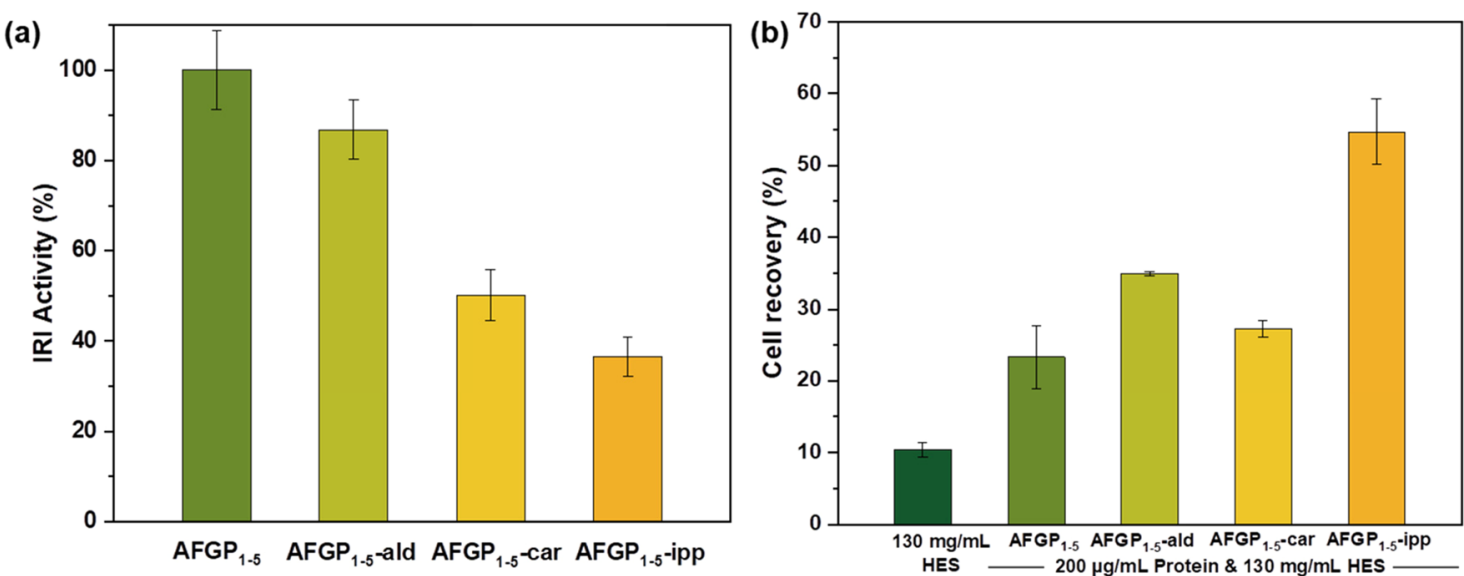

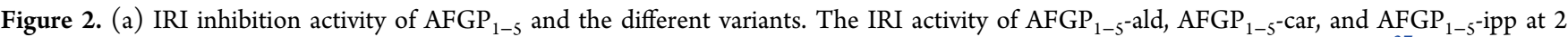
$\mu \mathrm{g} / \mathrm{mL}$ is reduced by $\sim 13,50$, and $63 \%$, respectively. Reprinted with permission from ref 37. Copyright 2021 ACS Publications. ${ }^{37}$ (b) Effects of $\mathrm{AFGP}_{1-5}$ and different variants on the cell recovery of cryopreserved hRBCs in HES solution $(130 \mathrm{mg} / \mathrm{mL})$, thawed at $23{ }^{\circ} \mathrm{C}$. Experiments were performed three times, and the error bars represent the standard deviation between the individual measurements.

The Abs (0\% hemolysis) and Abs (100\% hemolysis) represent the $414 \mathrm{~nm}$ absorbance values of the $0 \%$ and $100 \%$ hemolysis and control samples.

Preparation of the RBC Model Membrane. The phospholipids 1,2-dioleoyl-sn-glycero-3-phosphocholine (DOPC), N-palmitoyl-Derythro sphingosylphosphorylcholine (SM), and 1,2-dioleoyl-snglycero-3-phosphoethanolamine (DOPE) (Avanti Polar Lipids) were dissolved in chloroform/methanol $(9: 1$ mixture ( $\mathrm{vol} / \mathrm{vol}))$ at a ratio of 45:45:10 (mol \%). This composition was reported to serve as a phospholipid model of the outer leaflet of human RBC membranes. $^{38}$

Surface Pressure Measurements. Surface pressure measurements were performed in a custom-made Teflon trough filled with 5 $\mathrm{mL}$ of PBS buffer at room temperature $\left(22 \pm 1{ }^{\circ} \mathrm{C}\right)$ using a DeltaPi tensiometer (KBN 315 Sensor Head, Kibron Inc.). The surface pressure experiments were also performed under a nitrogen atmosphere to prevent the oxidation of unsaturated lipids. ${ }^{39,40}$ Surface pressure measurements were first performed at the air/buffer interface, and the phospholipid mixture solution of DOPC/SM/ DOPE was spread droplet by droplet at the air/ buffer interface using a Hamilton syringe. ${ }^{41,42} \mathrm{AFGP}_{1-5}(200 \mu \mathrm{L}), \mathrm{AFGP}_{1-5}$-ipp $(5 \mathrm{mg} / \mathrm{mL}$ in PBS), or pure buffer was injected into the subphase. The bulk AFGP concentration was $0.2 \mathrm{mg} / \mathrm{mL}$.

\section{RESULTS}

The cryopreservation of hRBCs was evaluated using a rapid freezing protocol, following experimental procedures described in established protocols. ${ }^{7,10}$ Samples were rapidly frozen by immersion in liquid nitrogen and subsequently stored at -196 ${ }^{\circ} \mathrm{C}$. Cell recoveries were determined after thawing at an ambient temperature of $23{ }^{\circ} \mathrm{C}$ or at $45^{\circ} \mathrm{C}$.

Figure 1a shows the results of the hRBC cryopreservation experiment in PBS buffer. The cell survival of hRBCs in PBS buffer was found to be low, with a recovery of $\sim 14 \%$. Upon the addition of $\mathrm{AFGP}_{1-5}$, the cell recovery decreased with increasing $\mathrm{AFGP}_{1-5}$ concentration. We found that for AFGP $_{1-5}$ concentrations exceeding $150 \mu \mathrm{g} / \mathrm{mL}$, the $\mathrm{hRBC}$ cell recovery was only $\sim 2 \%$. Clearly, the presence of AFGP ${ }_{1-5}$ in PBS buffer caused more damage than protection to the $\mathrm{hRBC}$. This finding is in line with the results from previous RBC cryopreservation studies using AFP type I and III. ${ }^{11,36}$ AFGPs can bind to ice crystals ${ }^{43}$ and shape small ice crystals to blunt hexagonal bipyramid crystals at a temperature slightly below its melting point. Upon lowering the temperature below the hysteresis gap, an ice crystal in the presence of AFGPs will rapidly grow into a bundle of spicular ice needles that likely damage the cells. ${ }^{44}$ During the rapid freezing and thawing process, which mimics the cryopreservation of RBCs, bipyramidal, ${ }^{11}$ needle-like, and specular ice crystals $^{45}$ were observed in the presence of AFPs, which damaged the RBCs and reduced cell survival significantly. Upon increasing the AFGP $_{1-5}$ concentrations in PBS buffer, this effect will become increasingly prominent, likely leading to the observed lower cell recovery of hRBCs.

Next, we investigated the capacity of $\mathrm{AFGP}_{1-5}$ to increase the survival of hRBC in HES solutions. We find that low (130 $\mathrm{mg} / \mathrm{mL}$ ) and high $(215 \mathrm{mg} / \mathrm{mL})$ concentrations of HES resulted in $\sim 12$ and $\sim 78 \%$ hRBC recovery (Figures $1 \mathrm{~b}$ and S1), respectively, and these cell recoveries are consistent with previous studies. ${ }^{10}$ Compared with $\mathrm{AFGP}_{1-5}$ in PBS buffer, which caused significant damage to hRBCs, AFGP $_{1-5}$ in HES solution exhibits much better protection owing to the suppression of the explosive ice growth beyond the $\mathrm{TH}$ gap. ${ }^{44}$ Even higher cell recovery rates can be obtained for AFGP $_{1-5}$ in high HES concentrations $(215 \mathrm{mg} / \mathrm{mL})$, but the very high HES concentration also leads to very viscous solutions with high osmotic pressure, which is impractical for clinical cryopreservation. ${ }^{44,46}$ In addition, transfusions containing high concentrations of HES have been shown to cause unwanted side effects like the inhibition of hemostasis in vulnerable patients. ${ }^{33}$ Therefore, the ability of AFGPs to modulate cellular recovery at low HES concentrations (130 $\mathrm{mg} / \mathrm{mL}$ ) will be investigated.

Figure $1 \mathrm{~b}$ shows the results of the cryopreservation experiment of $\mathrm{AFGP}_{1-5}$ in a $130 \mathrm{mg} / \mathrm{mL}$ HES solution. The effect of $\mathrm{AFGP}_{1-5}$ on the hRBC cryopreservation survival was again dependent on the $\mathrm{AFGP}_{1-5}$ concentration. We find that the cell recovery of hRBCs increased for concentrations up to $200 \mu \mathrm{g} / \mathrm{mL} \mathrm{AFGP}_{1-5}$ but decreased again at higher concentrations. The addition of $200 \mu \mathrm{g} / \mathrm{mL} \mathrm{AFGP}{ }_{1-5}$ showed the highest cell recovery $(\sim 24 \%)$ with twice the cell recovery of $130 \mathrm{mg} / \mathrm{mL}$ HES alone. The subsequent decrease in the cell recovery at higher concentrations agrees with observations for PVA, ${ }^{10}$ AFGP analogs, ${ }^{47}$ and AFP I. ${ }^{11}$

In order to determine whether there is a correlation between IRI activity and cryopreservation efficacy, three $\mathrm{AFGP}_{1-5}$ variants with different IRI and TH activities were investigated. 
In these variants, the hydroxyl groups of the galactose moieties of the natural $\mathrm{AFGP}_{1-5}$ isoforms were modified into $\mathrm{AFGP}_{1-5^{-}}$ aldehyde (AFGP ${ }_{1-5}$-ald), $\mathrm{AFGP}_{1-5}$-carboxyl (AFGP $1-5$-car), and $\mathrm{AFGP}_{1-5}$-isopropylidene $\left(\mathrm{AFGP}_{1-5}\right.$-ipp), as described recently. ${ }^{37,48}$ Because of the modifications, the IRI activities of $\mathrm{AFGP}_{1-5}$-ald, $\mathrm{AFGP}_{1-5}$-car, and $\mathrm{AFGP}_{1-5}$-ipp were reduced by 13,50 , and $63 \%$, respectively, relative to native $\mathrm{AFGP}_{1-5}$ (Figure 2a). ${ }^{37}$

We investigated the cryopreservation abilities of the variants at $200 \mu \mathrm{g} / \mathrm{mL}$ concentrations in HES $(130 \mathrm{mg} / \mathrm{mL})$ because these conditions showed the maximum cryopreservation efficiency for the native $\mathrm{AFGP}_{1-5}$. The results are shown in Figure 2b. Despite having lower IRI activity, the hRBC recovery of all three variants was increased. The cell recovery of $\mathrm{AFGP}_{1-5}$-ald and $\mathrm{AFGP}_{1-5}$-car was increased from $24 \%$ for $\mathrm{AFGP}_{1-5}$ to 35 and $27 \%$, while $\mathrm{AFGP}_{1-5}$-ipp more than doubled cell recovery to $55 \%$. Thus, despite having the lowest IRI activity ( $37 \%$ of native $\left.\mathrm{AFGP}_{1-5}\right)$, the $\mathrm{AFGP}_{1-5}$-ipp variant enabled the highest hRBC recovery, more than double that of native $\mathrm{AFGP}_{1-5}$.

Next, we determined the effects of five additional $\operatorname{AF}(G)$ Ps with varying IRI activities ${ }^{18}$ and compared their ability to improve the survival of hRBCs that were cryopreserved in 130 $\mathrm{mg} / \mathrm{mL}$ HES solutions (Figure 3 ). $\mathrm{AFGP}_{7-8}$ is an isoform and

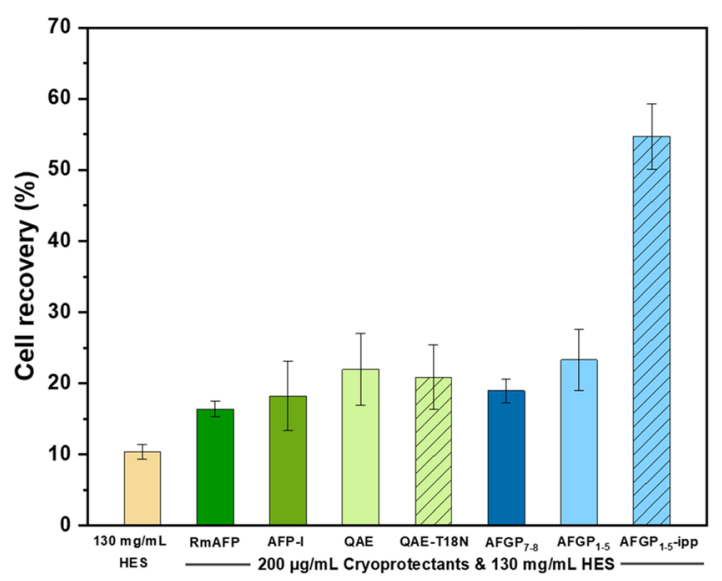

Figure 3. Effect of different $\mathrm{AF}(G)$ Ps and variants on the cell recovery of cryopreserved hRBC in HES solution $(130 \mathrm{mg} / \mathrm{mL})$, thawed at 23 ${ }^{\circ} \mathrm{C}$. Experiments were performed three times, and the error bars represent the standard deviation between the individual measurements.

low-molecular-weight variant of the AFGPs and possesses only $\sim 60 \%$ of the antifreeze activity of the larger $\mathrm{AFGP}_{1-5}$. 43,49 RmAFP is derived from the beetle Rhagium mordax and is considered to be a hyperactive AFP. ${ }^{14}$ QAE is a version of AFP type III with QAE-sephadex-binding, ${ }^{43,49}$ and AFP type I is a moderate AFP derived from winter flounder Pseudopleuronectes americanus. For the QAE mutant (QAE-T18N), the threonine 18 residue in the center of the ice-binding site was replaced by asparagine, causing a complete loss of $\mathrm{TH}$ activity ${ }^{50}$ and no effect on IRI activity. ${ }^{51}$ We find that the different $\operatorname{AF}(G) P s$ show remarkably similar cell recoveries $(\sim 20 \%)$ of hRBC despite displaying very different IRI activities (Figure S2). Interestingly, QAE-T18N has the same cell recovery as QAE, revealing that the $\mathrm{T} 18 \mathrm{~N}$ mutation and the associated loss of $\mathrm{TH}$ activity have little effect on the cryopreservation ability of hRBC. Of all investigated $\mathrm{AF}(G) \mathrm{Ps}$, the $\mathrm{AFGP}_{1-5}$-ipp variant shows the highest cell recovery of cryopreserved hRBC.
Apparently, the factors influencing the cryopreservation ability of an $\operatorname{AF}(G) \mathrm{P}$ are more complex and not solely dependent on IRI activity.

We performed surface pressure experiments of erythrocyte model membranes to further investigate the reason for AFGP $_{1-5}$-ipp's superior cryoprotective properties. Figure 4 shows the effect of $\mathrm{AFGP}_{1-5}$ and $\mathrm{AFGP}_{1-5}$-ipp on a model membrane mimicking the erythrocyte outer membrane, consisting of the phospholipid mixture DOPC, SM, and DOPE $(45: 45: 10(\mathrm{~mol} \%)) .^{38}$ The surface pressure of the model membrane in PBS buffer was set to $\sim 24 \mathrm{mN} / \mathrm{m}$, typical for a well-ordered, liquid-condensed model membrane. ${ }^{52}$ When proteins were injected under the monolayer, they are allowed to interact with the monolayer. If part of the protein inserts itself in between the lipid molecules or in any way disturbs the lipid packing, the surface pressure will increase. The increase hence suggests that the protein can interact with the monolayer. ${ }^{41}$ We find that the surface pressure increases substantially upon the addition of $\mathrm{AFGP}_{1-5}$ and $\mathrm{AFGP}_{1-5}$-ipp into the PBS subphase of the DOPC/SM/DOPE monolayer, as shown in Figure 4. This indicates that $\mathrm{AFGP}_{1-5}$ and $\mathrm{AFGP}_{1-5}$-ipp interact with the monolayer. $\Delta \pi$ represents the difference in the surface pressure after the addition of $\mathrm{AFGP}_{1-5} / \mathrm{AFGP}_{1-5}$-ipp and of the PBS buffer. The increase in surface pressure upon $\mathrm{AFGP}_{1-5} / \mathrm{AFGP}_{1-5}$-ipp injections suggests protein-membrane interactions and possible stabilization of the model membrane. We find the interaction of AFGP $_{1-5}$-ipp with the membrane to be stronger than that of $\mathrm{AFGP}_{1-5}$, as indicated by the $\Delta \pi$ of $\mathrm{AFGP}_{1-5}$-ipp, which is much higher than that for $\mathrm{AFGP}_{1-5}$ (Figure $4 \mathrm{a}$ ).

\section{DISCUSSION}

The cryopreservation of cells causes cold stress that affects the cell physiology, metabolic activity, and regulation of ion equilibration across membranes. The crystallization of water into ice is further detrimental to tissues, and the process of IRI upon thawing is the major contributor to cell death. We find that the addition of $\mathrm{AFGP}_{1-5}$ to a HES cryostorage medium has a statistically significant positive effect on the survival of hRBCs, which is consistent with previous studies on nonglycosylated AFPs. ${ }^{11,36}$ Different AFP classes with varying IRI activities showed very similar cell recovery of hRBC ( $\sim 20 \%)$, suggesting that IRI activity alone is not sufficient to predict cell recovery after cryopreservation. Interestingly, the chemically modified AFGP $_{1-5}$-ipp variant with additional hydrophobic groups on the galactose moieties promoted cell recovery of hRBC to $55 \%$ compared to the $24 \%$ of AFGP, while having decreased IRI and TH activity. Apparently, high IRI activity alone is insufficient to explain the beneficial effects of $\operatorname{AF}(G)$ Ps on hRBC survival after cryopreservation. Alternative mechanisms that explain the positive effect of $\mathrm{AF}(G)$ Ps on the cold survival of cells involve the protection of cell membranes as they pass through their phase transition temperatures as well as the blockage or alteration of the flow of ions into cells. Tomczak et al. proposed that $\operatorname{AF}(G) P s$ insert into membranes through hydrophobic interactions, thereby altering the molecular packing of the acyl chains, resulting in reduced membrane permeability and improved cryopreservation efficiency. ${ }^{53}$ We find that increasing AFGP hydrophobicity by adding an isopropylidene group leads to improved hRBC survival and stronger interaction with an erythrocyte model membrane. We hypothesize that the stabilization of the RBC membranes through AFGP and via hydrophobic interactions is 

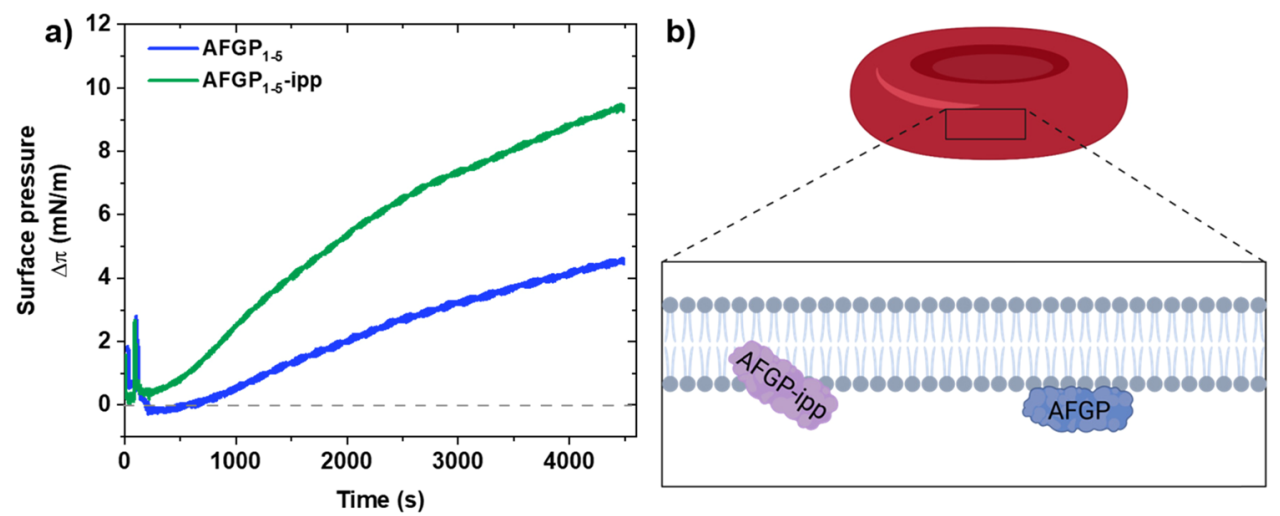

Figure 4. Interactions of $\mathrm{AFGP}_{1-5}$ with erythrocyte model membranes. (a) Difference in surface pressure $\Delta \pi$ versus time after the addition of $\mathrm{AFGP}_{1-5}$-ipp and $\mathrm{AFGP}_{1-5}$ into the PBS subphase of the DOPC/SM/DOPE monolayer. $\Delta \pi$ represents the change in the surface pressure after the addition of $\mathrm{AFGP}_{1-5}(5 \mathrm{mg} / \mathrm{mL})$ and of PBS buffer. (b) Schematic representation of $\mathrm{AFGP}_{1-5}$-ipp and $\mathrm{AFGP}_{1-5}$ proteins interacting with an $\mathrm{RBC}^{-}$ model membrane.

enhanced, owing to the modification. This modification substantially increases hRBC recovery. We suggest that apart from IRI activity, the capabilities to stabilize cell membranes and the cellular location of the cryoprotectants are additional key components for the cell recovery of hRBC after cryopreservation. However, membrane stabilization is an important but not the sole determining factor for cell survival after cryostorage. For example, bovine serum albumin is a membrane stabilizing agent, ${ }^{54}$ but it is usually used as a negative control for cryopreservation study. ${ }^{55}$ Apart from proteins, the lipids, sugars, and amino acids also seem to exert their cryoprotective effect at least partly by stabilizing the plasma membrane. ${ }^{54,56}$ Therefore, the combination of high IRI activity and capabilities to stabilize cell membranes is likely essential for optimizing cell survival after cryostorage.

\section{CONCLUSIONS}

Herein, we found a variety of antifreeze proteins that show similar cell recoveries of hRBCs despite having very different IRI activities. In addition, a chemically modified $\mathrm{AFGP}_{1-5}$-ipp variant with additional hydrophobic groups on the galactose moiety has decreased IRI activity but showed significantly increased cell recovery compared to $\mathrm{AFGP}_{1-5}$. Surface pressure experiments with the $\mathrm{AFGP}_{1-5}$-ipp variant showed enhanced interactions with $\mathrm{RBC}$ model membranes, indicating that membrane stabilization is another key factor in the survival of cells after cryostorage. We conclude that high IRI activity alone is insufficient to explain the beneficial effects of $\operatorname{AF}(G)$ Ps hRBC survival. The ability to stabilize cell membranes and the cellular location of the cryoprotectants are also likely crucial for cryopreservation and should be considered for the design of novel synthetic cryoprotectants.

\section{ASSOCIATED CONTENT}

\section{(5) Supporting Information}

The Supporting Information is available free of charge at https://pubs.acs.org/doi/10.1021/acs.biomac.1c01477.

Influence of different $\mathrm{AF}(G) \mathrm{Ps}$ on the cryopreservation of hRBC; IRI efficacy of various $\operatorname{AF}(G) P s$ and their variants (PDF)

\section{AUTHOR INFORMATION}

\section{Corresponding Author}

Konrad Meister - Max Planck Institute for Polymer Research, 55128 Mainz, Germany; University of Alaska Southeast, Juneau, Alaska 99801, United States; (1) orcid.org/00000002-6853-6325; Email: meisterk@mpip-mainz.mpg.de

\section{Authors}

Yuling Sun - Max Planck Institute for Polymer Research, 55128 Mainz, Germany; Institute of Chemistry, Chinese Academy of Sciences, Beijing 100190, P. R. China; (1) orcid.org/0000-0001-8582-5244

Daria Maltseva - Max Planck Institute for Polymer Research, 55128 Mainz, Germany

Jie Liu - Institute of Chemistry, Chinese Academy of Sciences, Beijing 100190, P. R. China

Theordore Hooker, II - University of Alaska Southeast, Juneau, Alaska 99801, United States

Volker Mailänder - Max Planck Institute for Polymer Research, 55128 Mainz, Germany; Dermatology Department, University Medical Center of the Johannes Gutenberg-University, 55131 Mainz, Germany

Hans Ramløv - Roskilde University, 4000 Roskilde, Denmark; @ orcid.org/0000-0003-1113-0583

Arthur L. DeVries - University of Illinois at Urbana-Champaign, Urbana, Illinois 61801, United States

Mischa Bonn - Max Planck Institute for Polymer Research, 55128 Mainz, Germany; (1) orcid.org/0000-0001-68518453

Complete contact information is available at:

https://pubs.acs.org/10.1021/acs.biomac.1c01477

\section{Funding}

Open access funded by Max Planck Society.

Notes

The authors declare no competing financial interest.

\section{ACKNOWLEDGMENTS}

K. Meister acknowledged support from BlaST, which is supported by the NIH Common Fund, through the Office of Strategic Coordination, Office of the NIH Director with the linked awards: TL4GM118992, RL5GM118990, and UL1GM118991. Research reported in this publication was also supported by an Institutional Development Award (IDeA) 
from the National Institute of General Medical Sciences of the National Institutes of Health under Grant No. 2P20GM103395. The content is solely the responsibility of the authors and does not necessarily represent the official view of the National Institutes of Health. Y.S. acknowledges support from the International Postdoctoral Exchange Fellowship Program of China. Figure ${ }^{4}$ and the TOC were created using BioRender.com.

\section{ABBREVIATIONS}

AFPs, antifreeze proteins; AFGPs, antifreeze glycoproteins; $\mathrm{AF}(G)$ Ps, antifreeze proteins and glycoproteins; hRBC, human red blood cell; $\mathrm{TH}$, thermal hysteresis; IRI, ice recrystallization inhibition; $\mathrm{AFGP}_{1-5}$-ipp, $\mathrm{AFGP}_{1-5}$-isopropylidene; $\mathrm{AFGP}_{1-5}$ ald, AFGP $_{1-5}$-aldehyde; AFGP ${ }_{1-5}$-car, AFGP ${ }_{1-5}$-carboxyl; PBS, phosphate-buffered saline buffer; HES, hydroxyethyl starch; DOPE, 1,2-dioleoyl-sn-glycero-3-phosphoethanolamine; DOPC, 1,2-dioleoyl-sn-glycero-3-phosphocholine; SM, Npalmitoyl-D-erythrosphingosylphosphorylcho-line

\section{REFERENCES}

(1) García-Roa, M.; del Carmen Vicente-Ayuso, M.; Bobes, A. M.; Pedraza, A. C.; González-Fernández, A.; Martín, M. P.; Sáez, I.; Seghatchian, J.; Gutiérrez, L. Red Blood Cell Storage Time and Transfusion: Current Practice, Concerns and Future Perspectives. Blood Transfus. 2017, 15, 222.

(2) Napolitano, L. M.; Kurek, S.; Luchette, F. A.; Anderson, G. L.; Bard, M. R.; Bromberg, W.; Chiu, W. C.; Cipolle, M. D.; Clancy, K. D.; Diebel, L. Clinical Practice Guideline: Red Blood Cell Transfusion in Adult Trauma and Critical Care. J. Trauma Acute Care Surg. 2009, 67, 1439-1442.

(3) Bohoněk, M., Cryopreservation of Blood. Blood transfusion in clinical practice; InTech Open, 2012; pp 233-242.

(4) Scott, K. L.; Lecak, J.; Acker, J. P. J. T. Biopreservation of Red Blood Cells: Past, Present, and Future. Transfus. Med. Rev. 2005, 19, 127-142.

(5) Wang, Z.; Yang, B.; Chen, Z.; Liu, D.; Jing, L.; Gao, C.; Li, J.; $\mathrm{He}$, Z.; Wang, J. Bioinspired Cryoprotectants of Glucose-Based Carbon Dots. ACS Appl. Bio Mater. 2020, 3, 3785-3791.

(6) Xue, B.; Zhao, L.; Qin, X.; Qin, M.; Lai, J.; Huang, W.; Lei, H.; Wang, J.; Wang, W.; Li, Y. Bioinspired Ice Growth Inhibitors Based on Self-Assembling Peptides. ACS Macro Lett. 2019, 8, 1383-1390.

(7) Bai, G.; Song, Z.; Geng, H.; Gao, D.; Liu, K.; Wu, S.; Rao, W.; Guo, L.; Wang, J. Oxidized Quasi-Carbon Nitride Quantum Dots Inhibit Ice Growth. Adv. Mater. 2017, 29, No. 1606843.

(8) Deller, R. C.; Vatish, M.; Mitchell, D. A.; Gibson, M. I. GlycerolFree Cryopreservation of Red Blood Cells Enabled by IceRecrystallization-Inhibiting Polymers. ACS Biomater. Sci. Eng. 2015, 1, 789-794.

(9) Lagerberg, J. W. Cryopreservation of Red Blood Cells. Methods Mol. Biol. 2015, 1257, 353-367.

(10) Deller, R. C.; Vatish, M.; Mitchell, D. A.; Gibson, M. I. J. N. Synthetic Polymers Enable Non-Vitreous Cellular Cryopreservation by Reducing Ice Crystal Growth During Thawing. Nat. Commun. 2014, 5, 3244.

(11) Carpenter, J. F.; Hansen, T. N. Antifreeze Protein Modulates Cell Survival During Cryopreservation: Mediation through Influence on Ice Crystal Growth. Proc. Natl. Acad. Sci. U. S. A. 1992, 89, 89538957.

(12) Meister, K.; Ebbinghaus, S.; Xu, Y.; Duman, J. G.; DeVries, A.; Gruebele, M.; Leitner, D. M.; Havenith, M. J. P. Long-Range Protein-Water Dynamics in Hyperactive Insect Antifreeze Proteins. Proc. Natl. Acad. Sci. U. S. A. 2013, 110, 1617-1622.

(13) DeVries, A. L.; Wohlschlag, D. E. Freezing Resistance in Some Antarctic Fishes. Science 1969, 163, 1073-1075.
(14) Meister, K.; Moll, C.; Chakraborty, S.; Jana, B.; DeVries, A.; Ramløv, H.; Bakker, H. Molecular Structure of a Hyperactive Antifreeze Protein Adsorbed to Ice. J. Chem. Phys. 2019, 150, 131101. (15) Knight, C. A.; De Vries, A. L.; Oolman, L. D. Fish Antifreeze Protein and the Freezing and Recrystallization of Ice. Nature 1984, 308, 295-296.

(16) Budke, C.; Dreyer, A.; Jaeger, J.; Gimpel, K.; Berkemeier, T.; Bonin, A. S.; Nagel, L.; Plattner, C.; DeVries, A. L.; Sewald, N.; Koop, T. Quantitative Efficacy Classification of Ice Recrystallization Inhibition Agents. Cryst. Growth Des. 2014, 14, 4285-4294.

(17) Kristiansen, E.; Zachariassen, K. E. The Mechanism by Which Fish Antifreeze Proteins Cause Thermal Hysteresis. Cryobiology 2005, $51,262-280$.

(18) Olijve, L. L. C.; Meister, K.; Devries, A. L.; Duman, J. G.; Guo, S.; Bakker, H. J.; Voets, I. K. Blocking Rapid Ice Crystal Growth through Nonbasal Plane Adsorption of Antifreeze Proteins. Proc. Natl. Acad. Sci. U. S. A. 2016, 113, 3740-3745.

(19) Geng, H.; Liu, X.; Shi, G.; Bai, G.; Ma, J.; Chen, J.; Wu, Z.; Song, Y.; Fang, H.; Wang, J. Graphene Oxide Restricts Growth and Recrystallization of Ice Crystals. Angew Chem., Int. Ed. Engl. 2017, 56, 997-1001.

(20) Zhu, W.; Guo, J.; Agola, J. O.; Croissant, J. G.; Wang, Z.; Shang, J.; Coker, E. N.; Motevalli, B.; Zimpel, A.; Wuttke, S. MetalOrganic Framework Nanoparticle-Assisted Cryopreservation of Red Blood Cells. J. Am. Chem. Soc. 2019, 141, 7789-7796.

(21) Allen, E. D.; Weatherbee, L.; Spencer, H.; Lindenauer, S. M.; Permoad, P. Large Unit Red Cell Cryopreservation with Hydroxyethyl Starch. Cryobiology 1976, 13, 500-506.

(22) Lionetti, F. J.; Hunt, S. M. Cryopreservation of Human Red Cells in Liquid Nitrogen with Hydroxyethyl Starch. Cryobiology 1975, 12, 110-118.

(23) Stiff, P. J.; Murgo, A. J.; Zaroulis, C. G.; DeRisi, M. F.; Clarkson, B. D. Unfractionated Human Marrow Cell Cryopreservation Using Dimethylsulfoxide and Hydroxyethyl Starch. Cryobiology 1983, 20, 17-24.

(24) Lapisson, G.; Salinas, C.; Malacher, P.; Michallet, M.; Philip, I.; Philip, T. Cryopreservation with Hydroxyethylstarch (Hes)+ Dimethylsulfoxide (Dmso) Gives Better Results Than Dmso Alone. Bull. Cancer 2004, 91, 10097-10102.

(25) Pasch, J.; Schiefer, A.; Heschel, I.; Rau, G. Cryopreservation of Keratinocytes in a Monolayer. Cryobiology 1999, 39, 158-168.

(26) Naaldijk, Y.; Johnson, A. A.; Friedrich-Stockigt, A.; Stolzing, A. Cryopreservation of Dermal Fibroblasts and Keratinocytes in Hydroxyethyl Starch-Based Cryoprotectants. BMC Biotechnol. 2016, $16,85$.

(27) Pasch, J.; Schiefer, A.; Heschel, I.; Dimoudis, N.; Rau, G. Variation of the Hes Concentration for the Cryopreservation of Keratinocytes in Suspensions and in Monolayers. Cryobiology 2000, 41, 89-96.

(28) Ashwood-Smith, M.; Warby, C.; Connor, K.; Becker, G. LowTemperature Preservation of Mammalian Cells in Tissue Culture with Polyvinylpyrrolidone (Pvp), Dextrans, and Hydroxyethyl Starch (Hes). Cryobiology 1972, 9, 441-449.

(29) Lionetti, F. J.; Hunt, S. M.; Gore, J. M.; Curby, W. A. Cryopreservation of Human Granulocytes. Cryobiology 1975, 12, 181-191.

(30) Waters, L.; Christensen, M.; Sato, R. Hetastarch: An Alternative Colloid in Burn Shock Management. J. Burn Care Rehabil. 1989, 10, 11-16.

(31) Stolzing, A.; Naaldijk, Y.; Fedorova, V.; Sethe, S. Hydroxyethylstarch in Cryopreservation-Mechanisms, Benefits and Problems. Transfus. Apher. Sci. 2012, 46, 137-147.

(32) Schmand, J. F.; Ayala, A.; Morrison, M. H.; Chaudry, I. H. Effects of Hydroxyethyl Starch after Trauma-Hemorrhagic Shock: Restoration of Macrophage Integrity and Prevention of Increased Circulating Interleukin-6 Levels. Crit. Care Med. 1995, 23, 806-814.

(33) Treib, J.; Baron, J.-F.; Grauer, M.; Strauss, R. An International View of Hydroxyethyl Starches. Intensive Care Med. 1999, 25, 258268. 
(34) Schramko, A. A.; Suojaranta-Ylinen, R. T.; Kuitunen, A. H.; Kukkonen, S. I.; Niemi, T. T. Rapidly Degradable Hydroxyethyl Starch Solutions Impair Blood Coagulation after Cardiac Surgery: A Prospective Randomized Trial. Anesth. Analg. 2009, 108, 30-36.

(35) Rubinsky, B.; Arav, A.; Devries, A. J. C. The Cryoprotective Effect of Antifreeze Glycopeptides from Antarctic Fishes. Cryobiology 1992, 29, 69-79.

(36) Chao, H.; Davies, P. L.; Carpenter, J. F. Effects of Antifreeze Proteins on Red Blood Cell Survival During Cryopreservation. Eur. J. Exp. Biol. 1996, 199, 2071-2076.

(37) Sun, Y.; Giubertoni, G.; Bakker, H. J.; Liu, J.; Wagner, M.; Ng, D. Y.; Devries, A. L.; Meister, K. Disaccharide Residues Are Required for Native Antifreeze Glycoprotein Activity. Biomacromolecules 2021, $22,2595-2603$

(38) Virtanen, J. A.; Cheng, K. H.; Somerharju, P. Phospholipid Composition of the Mammalian Red Cell Membrane Can Be Rationalized by a Superlattice Model. Proc. Natl. Acad. Sci. U. S. A. 1998, 95, 4964-4969.

(39) Liljeblad, J. F.; Bulone, V.; Tyrode, E.; Rutland, M. W.; Johnson, C. M. Phospholipid Monolayers Probed by Vibrational Sum Frequency Spectroscopy: Instability of Unsaturated Phospholipids. Biophys. J. 2010, 98, L50-L52.

(40) Inoue, K.-i.; Takada, C.; Wang, L.; Morita, A.; Ye, S. In Situ Monitoring of the Unsaturated Phospholipid Monolayer Oxidation in Ambient Air by Hd-Sfg Spectroscopy. J. Phys. Chem. B 2020, 124, 5246-5250.

(41) Brockman, H. Lipid Monolayers: Why Use Half a Membrane to Characterize Protein-Membrane Interactions? Curr. Opin. Struct. Biol. 1999, 9, 438-443.

(42) Eeman, M.; Deleu, M. From Biological Membranes to Biomimetic Model Membranes. Biotechnol., Agron., Soc. Environ. 2010, 14, 719-736.

(43) Meister, K.; DeVries, A. L.; Bakker, H. J.; Drori, R. Antifreeze Glycoproteins Bind Irreversibly to Ice. J. Am. Chem. Soc. 2018, 140, 9365-9368

(44) Liu, Z.; Wang, Y.; Zheng, X.; Jin, S.; Liu, S.; He, Z.; Xiang, J.-F.; Wang, J. Bioinspired Crowding Inhibits Explosive Ice Growth in Antifreeze Protein Solutions. Biomacromolecules 2021, 22, 26142624.

(45) Ishiguro, H.; Rubinsky, B. Mechanical Interactions between Ice Crystals and Red Blood Cells During Directional Solidification. Cryobiology 1994, 31, 483-500.

(46) Waymouth, C. Osmolality of Mammalian Blood and of Media for Culture of Mammalian Cells. In Vitro 1970, 6, 109-127.

(47) Matsumoto, S.; Matsusita, M.; Morita, T.; Kamachi, H.; Tsukiyama, S.; Furukawa, Y.; Koshida, S.; Tachibana, Y.; Nishimura, S.; Todo, S. Effects of Synthetic Antifreeze Glycoprotein Analogue on Islet Cell Survival and Function During Cryopreservation. Cryobiology 2006, 52, 90-98.

(48) Shier, W. T.; Lin, Y.; De Vries, A. L. Structure and Mode of Action of Glycoproteins from an Antarctic Fish. Biochim. Biophys. Acta 1972, 263, 406-413.

(49) Oude Vrielink, A. S.; Aloi, A.; Olijve, L. L.; Voets, I. K. J. B. Interaction of Ice Binding Proteins with Ice, Water and Ions. Biointerphases 2016, 11, No. 018906.

(50) Meister, K.; Strazdaite, S.; DeVries, A. L.; Lotze, S.; Olijve, L. L.; Voets, I. K.; Bakker, H. J. Observation of Ice-Like Water Layers at an Aqueous Protein Surface. Proc. Natl. Acad. Sci. U. S. A. 2014, 111, 17732-17736.

(51) Olijve, L. L. C.; Oude Vrielink, A. S.; Voets, I. K. A Simple and Quantitative Method to Evaluate Ice Recrystallization Kinetics Using the Circle Hough Transform Algorithm. Cryst. Growth Des. 2016, 16, 4190-4195.

(52) Roke, S.; Schins, J.; Müller, M.; Bonn, M. Vibrational Spectroscopic Investigation of the Phase Diagram of a Biomimetic Lipid Monolayer. Phys. Rev. Lett. 2003, 90, No. 128101.

(53) Tomczak, M. M.; Hincha, D. K.; Estrada, S. D.; Wolkers, W. F.; Crowe, L. M.; Feeney, R. E.; Tablin, F.; Crowe, J. H. A Mechanism

for Stabilization of Membranes at Low Temperatures by an Antifreeze Protein. Biophys. J. 2002, 82, 874-881.

(54) De Leeuw, F. E.; De Leeuw, A. M.; Den Daas, J. H. G.; Colenbrander, B.; Verkleij, A. J. Effects of Various Cryoprotective Agents and Membrane-Stabilizing Compounds on Bull Sperm Membrane Integrity after Cooling and Freezing. Cryobiology 1993, 30, 32-44.

(55) Izadyar, F.; Matthijs-Rijsenbilt, J. J.; Den Ouden, K.; Creemers, L. B.; Woelders, H.; de Rooij, D. G. Development of a Cryopreservation Protocol for Type a Spermatogonia. J. Androl. 2002, 23, 537-545.

(56) Crowe, J. H.; Crowe, L. M.; Carpenter, J. F.; Rudolph, A. S.; Wistrom, C. A.; Spargo, B. J.; Anchordoguy, T. J. Interactions of Sugars with Membranes. Biochim. Biophys. Acta, Biomembr. 1988, 947, 367-384.

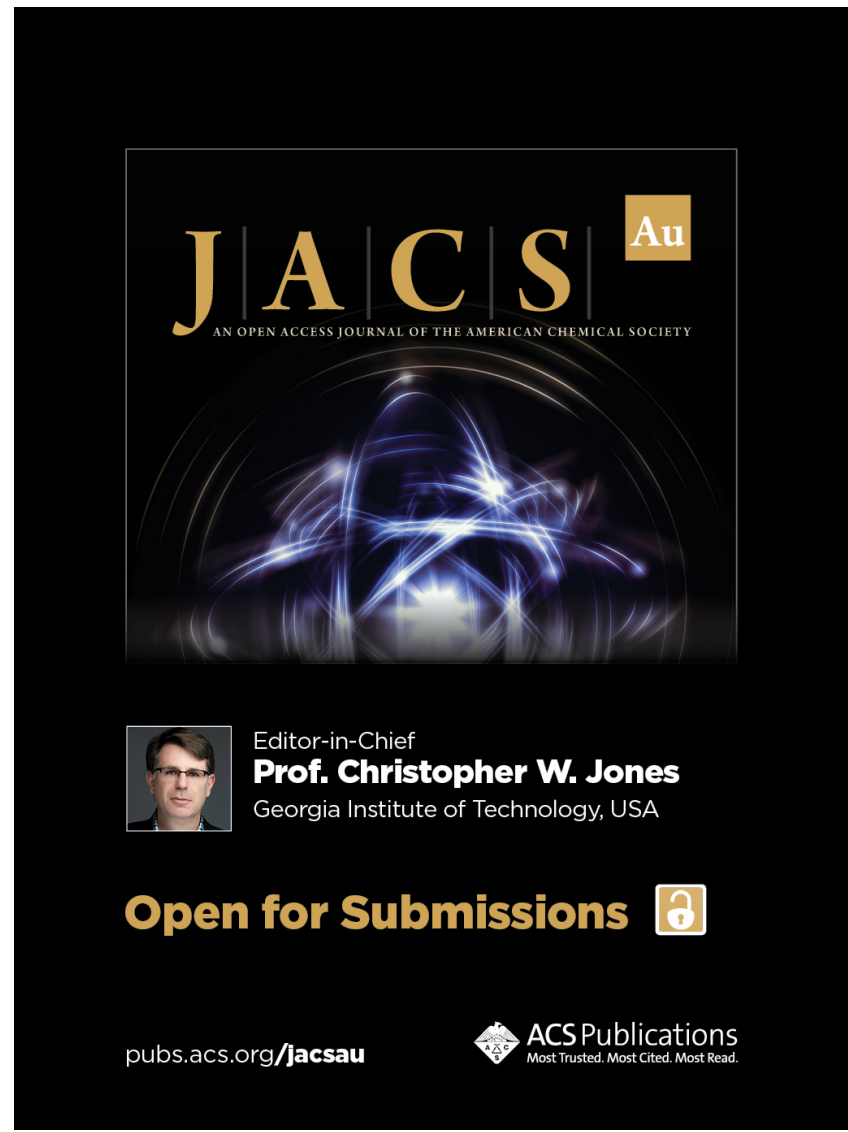

